\title{
THE ACTION GAMBLER AND EQUAL-SIZED WAGERING
}

\author{
DAVID HARTVIGSEN,* University of Notre Dame
}

\begin{abstract}
A gambler with an initial bankroll is faced with a finite sequence of identical and independent bets. For each bet, he may wager up to his current bankroll, and will win this amount with probability $p$ or lose it with probability $1-p$. His problem is to devise a wagering strategy that will maximize his final expected utility with the side condition that the total amount wagered (i.e. the total 'action') be at least his initial bankroll. Our main result is an expression that characterizes when the strategy of placing equal-sized wagers on all bets is optimal. In particular, for a given bankroll $B$, utility function $f$ (concave, increasing, differentiable), and $n$ bets, we show that it is optimal to wager $b / n$ on each bet if and only if the probability of winning each bet is less than or equal to some value $p^{*} \in\left[\frac{1}{2}, 1\right]$ (where $p^{*}$ is an explicit function of $B, f$, and $n$ ). We prove the result by using a basic nonlinear programming technique.
\end{abstract}

Keywords: Gambling; Kelly criterion; nonlinear programming

2000 Mathematics Subject Classification: Primary 91A60; 90C30; 60G40; 90C90

\section{Introduction}

Let us begin with an intuitive description of the general problem we consider. A gambler allocates an amount of money $B$ to a sequence of potential bets. The value $B$ is the minimum amount of 'action' or entertainment the gambler wants to purchase. That is, $B$ is the minimum total amount he wants to wager on these bets (as well as the maximum net amount he is willing to lose). The gambler's problem is to decide how much to wager on each bet.

More formally, we focus on the following model of this problem. A gambler allocates an amount of money $B$ (his initial bankroll) to a sequence of $n$ identical and independent bets. Each bet has two random outcomes: a win with probability $p$ or a loss with probability $1-p$. On the first bet in the sequence, the gambler can wager an amount up to $B$. His bankroll will increase or decrease by the amount of his wager, depending on whether he wins or loses the bet. On each subsequent bet, he can wager an amount up to his current bankroll. The value $B$ is the minimum amount of action or entertainment the gambler wants to purchase. That is, the total amount wagered on these $n$ bets must be at least $B$. We refer to this constraint as the action constraint and we refer to such a gambler as an action gambler. (By definition, $B$ is also the maximum net amount the gambler is willing to lose on these bets.) The action gambler has a concave (risk-averse), increasing, utility function for evaluating his final bankroll after the $n$ bets. A wagering strategy is a rule dictating how much the gambler should wager on each bet for any possible current bankroll. Our primary interest is the action gambler's problem, which is to determine a wagering strategy for the action gambler that maximizes the expected utility of the final bankroll.

Received 31 July 2008; revision received 21 January 2009.

* Postal address: 354 Mendoza College of Business, University of Notre Dame, Notre Dame, IN 46556-5646, USA.

Email address: dhartvig@nd.edu 
A simple strategy for the action gambler is to wager the same amount $B / n$ on each bet, which is sometimes referred to as a fixed stakes betting strategy. Our main result is an expression that characterizes when this strategy is optimal for the action gambler's problem. This result has the following form, for any concave, increasing, differentiable utility function $f$. Fixed stakes betting is optimal if and only if the probability $p$ of winning each bet is less than or equal to some value $p^{*} \in\left[\frac{1}{2}, 1\right]$, where $p^{*}$ is an explicit function of $B, f$, and $n$. Thus, fixed stakes betting is always optimal for $p \leq \frac{1}{2}$ (i.e. for fair and subfair bets), and it typically remains optimal for values of $p$ strictly greater than $\frac{1}{2}$ (i.e. for superfair bets).

We illustrate our main result by applying it to several common utility functions. For example, we show, for the utility function $f(y)=\log (a+y)$, where $a>0$, that it is optimal to always wager $B / n$ if and only if

$$
p \leq p^{*}=\frac{a+2 B}{2 a+4 B-2 B / n} .
$$

We see, in this and several other typical cases, that $p^{*} \rightarrow \frac{1}{2}$ (from above) as $n \rightarrow \infty$.

Another contribution of this paper is methodological. We prove the main result by formulating the action gambler's problem as a nonlinear program and then applying the wellknown Karush-Kuhn-Tucker conditions (see [14], [16] and, for example, [26, pp. 385-400]). Related gambling problems have previously been solved using different methods: e.g. dynamic programming or probabilistic techniques (see the discussion below). Because the formulation grows exponentially as $n$ grows, it quickly becomes impractical for finding optimal strategies when $p>p^{*}$ as $n$ grows. However, for small values of $n$, when $p>p^{*}$, the formulation can be used to find optimal wagering strategies, as we illustrate in Subsection 4.2. (For example, it could be used by an action gambler facing a small number of sports bets.) Furthermore, this formulation can be generalized in a number of ways (see the discussion below).

Before further discussing our results, let us put our problem into some context by considering several variations and the corresponding results from the literature.

Variation 1. This variation of the problem is the same as the action gambler's problem except that the $n$ bets are simultaneous. We call this the simultaneous betting problem. This is, of course, a variation on the (one-period) portfolio optimization problem, first studied in [19]. When the utility function is concave, increasing, and differentiable, then the expected utility is maximized by wagering $B / n$ on each bet for all values of $p$. (Intuitively, this result is what we would expect. Since the expected value of each bet is the same, all solutions have the same expected value. Hence, to obtain the optimal solution, we only need to minimize the risk, and this is accomplished by diversifying the portfolio as much as possible. We provide a proof of this result in Section 6. The proof is interesting since we apply the Karush-Kuhn-Tucker conditions to a formulation of the simultaneous betting problem that is closely related to the formulation we use for the action gambler's problem. Also, this result holds for a general class of utility functions.) Hence, our main result characterizes the case in which the optimal strategy for $n$ sequential bets is the same as the optimal strategy for $n$ simultaneous bets, when at least a total amount $B$ must be wagered.

Variation 2. This variation of the problem is the same as the action gambler's problem except that $n$ is unbounded, there is no action constraint, and the objective is to maximize the expected growth rate of the bankroll. The well-known optimal strategy, discovered by Kelly [15], is to always wager the fraction $2 p-1$ of the current bankroll when $p>\frac{1}{2}$ and to wager nothing when $p \leq \frac{1}{2}$. We will discuss (in Section 3) how, when $p>\frac{1}{2}$, the action constraint is met by 
this solution in the limit as $n \rightarrow \infty$. An interesting feature of this strategy, when $p>\frac{1}{2}$, is that it is equivalent to maximizing a log utility function for each bet.

Variation 3. This variation of the problem is the same as the action gambler's problem except that we remove the action constraint. In this case the problem has been solved for all values of $p$ in [1] for the special cases of the logarithmic $(f(y)=\log (y))$ and power $\left(f(y)=y^{\alpha}, 0<\right.$ $\alpha<1)$ utility functions, where it is optimal to wager nothing if $p \leq \frac{1}{2}$ and, otherwise, it is optimal to wager a fixed fraction of the current bankroll. (Guu and Wang [12] found a complete characterization of the utility functions for which the optimal solution is to wager a fixed fraction.) This problem has also been solved by Cetinkaya and Parlar [3] for the $\log (a+y)$ utility function $(a>0)$, where the optimal solution depends on $p$ and, for each bet $j$, the size of the current bankroll and the number $j$. These variations are solved using dynamic programming techniques. Typically, optimal solutions to the unconstrained problem are not feasible for the constrained problem (see the discussion below). However, for the utility function $f(y)=\log (a+y)$, it is shown in [3] that, for sufficiently large $p$, it is optimal to wager the entire bankroll on the first bet, in which case the action constraint is satisfied. As for Variation 2, solutions for the unconstrained problem can approach feasibility for the constrained problem as $n$ grows.

Variation 4. This variation of the problem is the same as the action gambler's problem except that the minimum total amount the gambler wants to wager is $A$, where $A \leq B$. Thus, $B$ is still the maximum net amount the gambler is willing to lose. We call this the general action gambler's problem. In this paper we characterize the case in which the fixed stakes strategy of wagering $A / n$ on all bets is optimal for this problem. (Observe that if an action gambler wins a bet before the final bet then he faces a general action gambler's problem on the remaining bets because the remaining amount that must be wagered is strictly less than the current bankroll.)

Let us briefly emphasize how our results differ from those discussed for Variation 3 above. The results for Variation 3 are for specific utility functions, while our results hold for a large, general class of utility functions. On the other hand, the results for Variation 3 hold for all $p$, whereas our results hold for a range of values of the form $p \leq \frac{1}{2}+\varepsilon$ (for $\varepsilon \geq 0$ ). (Of course, values of $p$ in such a range are common.)

The next difference between our results and those of Variation 3 is a main motivation for this paper. When $n$ is small and/or $p \leq \frac{1}{2}+\varepsilon$ (for small $\varepsilon>0$ ), the optimal strategies for the unconstrained problems considered in Variation 3 can have the gambler wagering a total amount significantly less than $B$. (Observe that, for $p \leq \frac{1}{2}$, a common situation, the optimal unconstrained wager is always 0 for all $n$. Furthermore, a small value of $n$ with $p \leq \frac{1}{2}+\varepsilon$ (for small $\varepsilon>0$ ) captures the common situation faced by a weekend sports gambler. We consider examples of this type in more detail in Subsection 4.2.) Such solutions seem unrealistic for those who view gambling as a form of entertainment of which they want to purchase a certain amount. Furthermore, if a significant fraction (say one third) of the bankroll is never at risk then the function of the bankroll in the model seems questionable. To elaborate, suppose a person, using a bankroll $B$ and an optimal unconstrained strategy, cannot lose more than, say, two thirds of $B$. This person could, theoretically, allocate a larger bankroll $B^{\prime}$ to the sequence of bets and still never risk losing, under optimal play for $B^{\prime}$, an amount equal to the original bankroll $B$; but the optimal strategies calculated using $B$ and $B^{\prime}$ could be different. (It is easy to construct such examples based on the discussion in Section 3.) Observe that the same situation can occur for the generalized action gambler's problem when $A<B$ and it is optimal to wager $A / n$ on every bet. For this reason, we present the more general characterization theorem in a 
separate section of the paper and its proof is given as a variation on the proof of the main result for the action gambler's problem.

A final difference between our results and those of Variations 2 and 3, as mentioned above, is the methodology we use to prove our results. Probabilistic techniques are used to prove the Variation 2 results and dynamic programming techniques are used to prove the Variation 3 results. In this paper we make use of nonlinear programming techniques.

Let us point out another feature of this paper's nonlinear programming approach. The basic model can easily be generalized to explore, for example, the following variations of the action gambler's problem (and its unconstrained variation).

- Allow bets to be both sequential and simultaneous.

- Allow bets to take multiple periods to be resolved.

- Allow bets to have general payoffs.

- Allow bets to have three or more outcomes.

- Allow bets to have minimum wager sizes.

- Do not require all bets to be identical. (Thus, we could have combinations of bets of the types listed above.)

There is a large literature on gambling problems similar to the one considered here. A classic work is the book by Dubins and Savage [6]. It focuses on a different objective function for the unconstrained problem, when $p \leq \frac{1}{2}$ : maximizing the probability of reaching a target final bankroll, where the number of sequential bets is unbounded. The same problem, when $p>\frac{1}{2}$ (where the number of bets is both bounded and unbounded), was first studied by Breiman [2]. Related work (based on discrete-time models and often with side constraints added to the basic models discussed above) includes the following: [4], [5, pp. 391-394], [7]-[11], [13], [17], [20], [22]-[25], and [28].

This paper is organized as follows. Section 2 contains the statements of the main results and some applications of the results using four common utility functions. In Section 3 we show how solutions to some unconstrained problems in Variation 3 (above) can be 'very infeasible' for the action gambler's problem. This phenomenon is one of the motivations behind this paper. The nonlinear programming formulation of the action gambler's problem is presented in Section 4, where it is used to directly solve some small examples. In Section 5 we present the version of the main theorem for the generalized action gambler's problem. Section 6 contains proofs of the main results, and in Section 7 we suggest an open problem.

\section{Main results}

In this section we present our main theorem, which characterizes when it is optimal to wager $B / n$ on all bets for the action gambler's problem for a large class of utility functions. We also present a related proposition that refines the characterization for a smaller class of well-known utility functions. We illustrate these results with four examples of commonly studied utility functions.

As described in the introduction, we have $n$ bets that occur in sequence. Each bet has a probability $p$ of being a win and a probability $1-p$ of being a loss. The gambler begins with an initial bankroll of size $B>0$ and can wager any amount of his current bankroll on a bet. 
He either wins or loses the amount of his wager on a bet. The total amount wagered on the $n$ bets must be at least $B$.

Let us observe that the range of final bankrolls for our gambler is $[0, M]$, where $M \equiv B 2^{n}$. (Here $M$ is the final bankroll corresponding to $n$ wagers, each of the entire current bankroll, and $n$ wins.)

Next we state our main result, which characterizes when a $B / n$ fixed stake strategy is optimal for a large class of utility functions.

Theorem 1. Let $f(y)$ be a concave, increasing, and differentiable utility function on $[0, M]$, and let $n \geq 2$. Then, for the action gambler's problem, wagering $B / n$ on each bet maximizes the expected utility of the final bankroll if and only if

$$
p \leq \min _{\omega \in\{2, \ldots, n\}}\left(1+\frac{\mathrm{d}}{\mathrm{d} \omega} f\left(\frac{2 B}{n} \omega\right) / \frac{\mathrm{d}}{\mathrm{d} \omega} f\left(\frac{2 B}{n}(\omega-1)\right)\right)^{-1} .
$$

When $n=1$, the unique feasible solution is to wager $B$ for all values of $p$.

Remark 1. Because $f(y)$ is increasing and differentiable on $[0, M]$, the minimization is well defined; in particular,

$$
\frac{\mathrm{d}}{\mathrm{d} \omega} f\left(\frac{2 B}{n}(\omega-1)\right)>0 \text { for } \omega \in\{2, \ldots, n\} .
$$

Remark 2. It is well known that if a function $f$ is concave and differentiable on an interval, then the derivative of $f$ is nonincreasing on the interval (see, e.g. [21, p. 142]). Therefore, the bound on $p$ in (1) is in $\left[\frac{1}{2}, 1\right]$.

For contrast, we now state a related result for the simultaneous betting problem (Variation 1 in the introduction). A proof of this result appears in Section 2.

Theorem 2. Let $f(y)$ be a concave and differentiable utility function on $[0,2 B]$. Then, for the simultaneous betting problem, wagering $B / n$ on each bet maximizes the expected utility of the final bankroll (for all values of $p$ ).

Theorem 2 allows us to make the following remark.

Remark 3. Let $f(y)$ be a concave and differentiable utility function on $[0,2 B]$, and let $m>n$. Then the expected utility of wagering $B / m$ on $m$ sequential bets is greater than or equal to the expected utility of wagering $B / n$ on $n$ sequential bets (since wagering $B / n$ on $n$ of the $m$ bets is feasible, and by treating the bets as simultaneous and applying Theorem 2 ). (We assume that the individual bets are the same in both situations.) Hence, if the action gambler is using a fixed stakes strategy, it is preferable for him to spread his wagering over as many bets as possible. However, if it is optimal to wager $B / n$ on $n$ sequential bets then it is not necessarily optimal to wager $B / m$ on $m$ sequential bets (see Remark 4, below).

It turns out that, for some standard classes of utility functions, we can state which value of $\omega$ determines the bound on $p$ in (1).

Let $f(y)$ be a concave, increasing, twice differentiable utility function on some interval $[c, d]$. Then the Arrow-Pratt absolute risk aversion coefficient is defined on $[c, d]$ as follows:

$$
h(y)=-\frac{f^{\prime \prime}(y)}{f^{\prime}(y)} .
$$


We say that a person's utility has increasing, decreasing, or constant absolute risk aversion over $[c, d]$ if $h(y)$ is increasing, decreasing, or, respectively, constant as $y$ increases over $[c, d]$. (These notions of risk aversion are well known; see, e.g. [18, p. 233]). We prove the following result in Subsection 6.3.

Proposition 1. Let $f(y)$ be a concave, increasing, twice differentiable function on $[0, M]$, and let $n \geq 2$. Then, for the action gambler's problem,

- the bound on $p$ in (1) is minimized at $\omega=2$ if $f$ has increasing absolute risk aversion over $[2 B / n, 2 B]$;

- the bound on $p$ in (1) is minimized at $\omega=n$ if $f$ has decreasing absolute risk aversion over $[2 B / n, 2 B]$;

- the bound on $p$ in (1) is minimized at $\omega=2, \ldots, n$ if $f$ has constant absolute risk aversion over $[2 B / n, 2 B]$.

Next we look at four examples of commonly studied utility functions. We assume that $n \geq 2$. The assumptions of Proposition 1 apply in each case and each of the three types of risk aversion is exhibited.

Example 1. Let $f(y)=\log (a+y)$ for $a>0$. Since $h(y)=1 /(a+y)^{2}$, $f$ has decreasing absolute risk aversion. Hence, the bound on $p$ in (1) is minimized at $\omega=n$, and we see that it is optimal to wager $B / n$ if and only if

$$
p \leq \frac{a+2 B}{2 a+4 B-2 B / n} .
$$

For instance, if $B=1000, a=10$, and $n=3$, we obtain $p \leq 0.60$.

Example 2. Let $f(y)=y^{\alpha}$ for $0<\alpha<1$. Since $h(y)=(1-\alpha) / y$, $f$ has decreasing absolute risk aversion. Hence, the bound on $p$ in (1) is minimized at $\omega=n$, and we see that it is optimal to wager $B / n$ if and only if

$$
p \leq \frac{1}{1+((n-1) / n)^{1-\alpha}} .
$$

For instance, if $\alpha=0.5$ and $n=3$, we obtain $p \leq 0.55$.

Example 3. Let $f(y)=-\mathrm{e}^{-b y}$ for $b>0$. Since $h(y)=b, f$ has constant absolute risk aversion. Hence, the bound on $p$ in (1) is the same at all $\omega=2, \ldots, n$, and we see that it is optimal to wager $B / n$ if and only if

$$
p \leq \frac{1}{1+\mathrm{e}^{-2 B b / n}} .
$$

For instance, if $B=1000, b=0.001$, and $n=3$, we obtain $p \leq 0.66$.

Example 4. Let $f(y)=y-c y^{2}$ for $0<c \leq 1 / 2 M$. (Note that $f$ is maximized at $y=1 / 2 c$; hence, we require that $1 / 2 c \geq M$ in order for the utility function to be increasing over $[0, M]$.) Since $h(y)=1 /(1 / 2 c-y), f$ has increasing absolute risk aversion (for $c \leq 1 / 2 M$ ). Hence, the bound on $p$ in (1) is minimized at $\omega=2$, and we see that it is optimal to wager $B / n$ if and only if

$$
p \leq \frac{1-4 B c / n}{2-12 B c / n} .
$$

For instance, if $B=1000, c=0.00005$, and $n=3$, we obtain $p \leq 0.52$. 
Observe that, in Examples 1-3, the assumptions on $f$ continue to hold as $n$ increases; hence, the formulae demonstrate the following remark. Let $p^{*}$ denote the upper bound in (1).

Remark 4. In Examples 1-3, $p^{*} \rightarrow \frac{1}{2}$ (from above) as $n \rightarrow \infty$.

The property in Remark 4 is not implied by the formula of Example 4 (although it looks that way), because, as $n$ increases, so does $M$; hence, at some point, $f$ will no longer be increasing on $[0, M]$ (i.e. the assumption on $c$ will eventually fail as $n$ increases). However, for all four examples, we can make the following remark when the number of bets is smaller than $n$.

Remark 5. In Examples 1-4, if it is optimal to wager $B / n$ on each of the $n$ bets then it will be optimal to wager $B / m$ on each of the $m$ bets when $m<n$.

\section{Context}

In this section we compare solutions of the constrained problem (the action gambler's problem) to solutions of some previously studied special cases of the unconstrained problem (Variation 3 in the introduction). We find that in common situations, the total amount wagered using optimal solutions for the unconstrained problem can be significantly less than $B$; hence, significantly infeasible for the constrained problem. This was part of the motivation for studying the action gambler's problem.

Let us consider a solution strategy that would have us always wager a fixed fraction $r$, where $0 \leq r \leq 1$, of the current bankroll. (We consider two such examples below.) We have the following simple proposition.

Proposition 2. Suppose that we always wager a fraction $r$, where $0 \leq r \leq 1$, of the current bankroll. Then the minimum total amount wagered is equal to $B\left[1-(1-r)^{n-1}\right]$, which occurs when the first $n-1$ bets are lost; and the maximum total amount wagered is equal to $B\left[(1+r)^{n-1}-1\right]$, which occurs when the first $n-1$ bets are won.

Proof. Observe that the amount wagered on bet $i$ is $r(1+r)^{j}(1-r)^{k} B$, where $j$ equals the number of bets won before bet $i, k$ equals the number of bets lost before bet $i$, and $j+k=i-1$. Clearly, this is a minimum when $j=0$ and a maximum when $k=0$. Hence (using the formulae for the sum of a geometric series when $r>0$ ), the total amount wagered reaches its minimum when the first $n-1$ bets are lost and this is equal to

$$
r B \sum_{i=0}^{n-2}(1-r)^{i}=r B \frac{1-(1-r)^{n-1}}{1-(1-r)}=B\left[1-(1-r)^{n-1}\right] .
$$

Similarly, the total amount wagered is a maximum when the first $n-1$ bets are won and this is equal to

$$
r B \sum_{i=0}^{n-2}(1+r)^{i}=r B \frac{1-(1+r)^{n-1}}{1-(1+r)}=B\left[(1+r)^{n-1}-1\right] .
$$

Observe that the simplified formulae are also correct when $r=0$ (where the total amount wagered is 0 ). This completes the proof.

Let us make some general observations based on Proposition 2. First, we see that, for all values of $r<1$ and all values of $n$, a fixed fraction solution is infeasible for our constrained problem since the minimum total amount wagered is strictly less than $B$. In particular, when $r$ is 'small' and $n$ is 'not too large', the fixed fraction solution can be 'very infeasible', in the 
sense that the minimum total amount wagered can be 'significantly' less than $B$ and even the maximum total amount wagered can be strictly less than $B$ (see the examples below).

Another observation is that, for all values of $r<1$, a fixed fraction solution gets arbitrarily close to being feasible for our constrained problem, as $n \rightarrow \infty$. However, wagers for the fixed fraction solution can become arbitrarily small as $n$ grows. In particular, for a fixed fraction solution, the final wager along the all-losing path is $r B(1-r)^{n-1}$, while, for the fixed stakes solution, the final wager is $B / n$. These amounts can be quite different (and the required wager for the fixed fraction strategy is much more likely to be unplayable in a real situation).

Next we expand the above discussion by considering two wagering strategies, due to Bellman and Kabala [1], that involve fixed fraction strategies for the unconstrained problem. They showed that fixed fraction strategies are optimal for the utility functions $f(y)=\log (y)$ and $f(y)=y^{\alpha}$ for $0<\alpha<1$. In particular, for $f(y)=\log (y)$, it is optimal to always wager the fraction $r=2 p-1$ when $p>\frac{1}{2}$ and to wager 0 when $p \leq \frac{1}{2}$. (Kelly [15] showed that this strategy is also optimal for the objective of maximizing the expected growth rate of the bankroll as $n \rightarrow \infty$.) For $f(y)=y^{\alpha}$, it is straightforward to calculate that it is optimal to always wager the fraction

$$
r=\frac{(p /(1-p))^{1 /(1-a)}-1}{(p /(1-p))^{1 /(1-a)}+1} \quad \text { when } p>\frac{1}{2}
$$

and to wager 0 when $p \leq \frac{1}{2}$. Finally, let us remark that the optimal solution for $f(y)=$ $\log (a+y)$ is not typically a fixed fraction when $p>\frac{1}{2}$ (see [3]), although, as $a \rightarrow 0$, the solution approaches the solution given above for $f(y)=\log (y)$; when $p \leq \frac{1}{2}$, it is again optimal to wager 0 .

Clearly, when $p \leq \frac{1}{2}$, these examples result in solutions that are far from feasible for the constrained problem. As we illustrate next, this can also be true for values of $p$ slightly above $\frac{1}{2}$ and for values of $n$ that are not too large.

Let us consider two detailed examples. Let $p^{*}$ denote the appropriate upper bound in (1). Suppose that we set $p=0.55, B=1000$, and $n=3$; let $a$ be very close to 0 , and let $\alpha=0.5$. For $f(y)=\log (a+y)$, we have $r \approx 2 p-1=0.1$ and $p^{*} \approx 0.6$. Hence, in the unconstrained case, Proposition 2 shows that our total wager is, approximately, between 190 and 210. For $f(y)=y^{\alpha}$, we have $r \approx 0.2$ and $p^{*} \approx 0.55$. Hence, in the unconstrained case, Proposition 2 tells us that our total wager is, approximately, between 360 and 440. In both examples, in the constrained case, we wager 333.33 on each bet, since $p \leq p^{*}$. (Observe that our result for the constrained problem (Theorem 1) does not hold for $f(y)=\log (y)$ because it is not differentiable at 0 ; this is why we have considered $f(y)=\log (a+y)$ for small $a$.)

\section{The formulation and some examples}

In this section we first introduce some notation and give our nonlinear programming formulation for the action gambler's problem, when $n \geq 2$. Then we use the formulation to find optimal strategies for a few small-size examples in order to examine the structure of the solutions and to compare them with the main result in Theorem 1.

\subsection{Notation and formulation}

Our first step is to define a directed binary tree that describes all possible outcomes of the $n$ bets. (See Figure 1 for an example with $n=3$.) Let $T=(V, A)$ be a directed tree with $|V|=2^{n+1}-1$. The node set $V$ is partitioned into two sets: Leaves $(T)$ and $\operatorname{NonLeaves}(T)$, where $|\operatorname{Leaves}(T)|=2^{n}$. There is one special node $r \in \operatorname{NonLeaves}(T)$ called the root of $T$. 


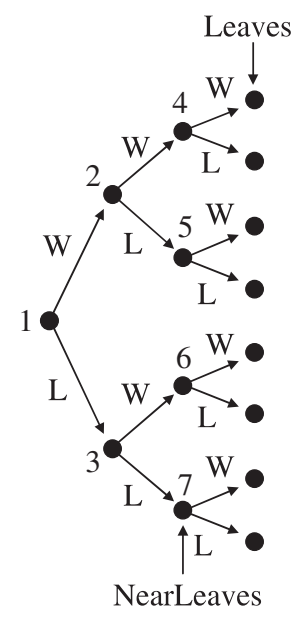

FIGURE 1: A directed binary tree.

The tree $T$ must satisfy the following properties.

- The outdegree of each node in $\operatorname{NonLeaves}(T)$ is 2 .

- The outdegree of each node in $\operatorname{Leaves}(T)$ is 0 .

- The indegree of each node in $V \backslash r$ is 1 .

- The indegree of $r$ is 0 .

- For each $v \in \operatorname{Leaves}(T)$, the number of arcs on the directed path from $r$ to $v$ is $n$.

In Figure 1, the node labeled 1 is the root and the eight nodes on the far right are Leaves $(T)$. For each pair of arcs of the form $u v, u v^{\prime}$, one arc is labeled $\mathrm{W}$ (for win) and one is labeled $\mathrm{L}$ (for loss). We use the following additional terminology.

- NearLeaves $(T)$ denotes the subset of $\operatorname{NonLeaves}(T)$ that are adjacent to a node in Leaves $(T)$. (In Figure 1, NearLeaves $(T)=\{4,5,6,7\}$.)

For each node $v \in V$, we introduce the following definitions.

- $\operatorname{RootPath}(v)$ denotes the directed path from $r$ to $v$.

- Leaves $(v)$ denotes the subset of Leaves $(T)$ reachable from $v$ by a directed path. (In Figure 1, Leaves $(2)=$ the uppermost four leaves.)

- $w(v)$ denotes the number of $\operatorname{arcs}$ labeled $\mathrm{W}$ on RootPath $(v)$. (In Figure 1, w(5) $=1$.)

- $l(v)$ denotes the number of $\operatorname{arcs}$ labeled L on $\operatorname{RootPath}(v)$.

For each node $v \in \operatorname{NonLeaves}(T)$, we define

- NearLeaves $(v)$ to be the subset of NearLeaves $(T)$ reachable from $v$ by a directed path. (In Figure 1, NearLeaves $(2)=\{4,5\}$.)

If $u v \in A$ then $\operatorname{Pred}(v)=u$, i.e. $u$ is the predecessor of $v$. 
Each node $v$ in NonLeaves $(T)$ represents a bet with a unique history determined by $\operatorname{RootPath}(v)$. The bet is the number of $\operatorname{arcs}$ in $\operatorname{RootPath}(v)$ plus 1 and the sequence of wins and losses on this path represents the history. For example, in Figure 1, the root node 1 represents bet 1 ; nodes 2 and 3 represent bet 2 (preceded by a win and a loss on the first bet, respectively); and nodes 4, 5, 6, and 7, represent bet 3 (preceded by (win, win), (win, loss), (loss, win), and (loss, loss) on the first two bets, respectively). The nodes in Leaves $(T)$ represent the final outcome of the betting.

We let $x \in \mathbb{R}^{\text {NonLeaves(T) }}$ denote a variable vector. The values of the components of $x$ are the amounts we wager in each situation corresponding to a node in $\operatorname{NonLeaves}(T)$. For each $v \in V$, we let $d^{v} \in \mathbb{R}^{\operatorname{NonLeaves}(T)}$ denote a vector defined as follows:

$$
d_{i}^{v}= \begin{cases}+1 & \text { if node } i \text { is on } \operatorname{RootPath}(v), i \neq v, \text { and the arc out of } i \\ & \text { on } \operatorname{RootPath}(v) \text { is a W; } \\ -1 & \text { if node } i \text { is on } \operatorname{RootPath}(v), i \neq v, \text { and the arc out of } i \\ & \text { on } \operatorname{RootPath}(v) \text { is an L; } \\ 0 \quad \text { otherwise. }\end{cases}
$$

Observe that $d^{r}=0$.

For each $v \in$ NearLeaves $(T)$, we let $e^{v} \in \mathbb{R}^{\text {NonLeaves(T) }}$ denote a vector defined as follows:

$$
e_{i}^{v}= \begin{cases}+1 & \text { if node } i \text { is on } \operatorname{RootPath}(v) \\ 0 & \text { otherwise }\end{cases}
$$

We can now formulate the action gambler's problem as a nonlinear program as follows, where $T$ denotes the directed binary tree corresponding to the $n$ bets:

$$
\max \sum_{v \in \operatorname{Leaves}(T)} p^{w(v)}(1-p)^{l(v)} f\left(B+d^{v} x\right)
$$

such that

$$
\begin{gathered}
x_{v} \leq B+d^{v} x \quad \text { for all } v \in \operatorname{NonLeaves}(T), \\
e^{v} x \geq B \quad \text { for all } v \in \operatorname{NearLeaves}(T), \\
x \geq 0
\end{gathered}
$$

The objective function is simply the sum of the utilities of the possible final bankrolls, weighted by their probabilities. The constraints in (2) say that the gambler cannot wager more than his remaining bankroll on any bet. The constraints in (3) say that the gambler must always wager at least $B$, in total.

Next we rewrite the above system, which will be useful in the proof of our main result. Let $v^{L}$ denote the node in NearLeaves $(T)$ such that $w\left(v^{L}\right)=0$ and $l\left(v^{L}\right)=n-1$. Observe that, for $v^{L}$, the corresponding inequalities in constraints (2) and (3) are identical, except that the inequality signs are in opposite directions. Hence, the nonlinear program can be rewritten (by replacing constraints (2) and (3) with constraints (4), (5), and (6), below) as follows:

$$
\max \sum_{v \in \operatorname{Leaves}(T)} p^{w(v)}(1-p)^{l(v)} f\left(B+d^{v} x\right)
$$


such that

$$
\begin{gathered}
x_{v} \leq B+d^{v} x \quad \text { for all } v \in \operatorname{NonLeaves}(T) \backslash v^{L}, \\
e^{v} x \geq B \quad \text { for all } v \in \operatorname{NearLeaves}(T) \backslash v^{L}, \\
e^{v^{L}} x=B, \\
x \geq 0 .
\end{gathered}
$$

\subsection{Examples}

Tables 1 and 2 contain optimal solutions to the action gambler's problem using the parameters of the four examples in Section 2 and various values of $p$. We consider $n=2$ and $n=3$, and use the indices on $x$ from Figure 1. The constrained nonlinear optimizer in MATHEMATICA ${ }^{\circledR}$, version 6 [27] was used to find these solutions using the formulation in the previous section.

Observations. (i) The wagers are equal to $B / n$ whenever $p$ is less than or equal to the bounds given in the examples in Section 2.

(ii) The utility function $f(y)=y-c y^{2}$ shows that it is sometimes optimal (for large $p$ ) to always wager the entire current bankroll.

TABLE 1: Optimal solutions to the action gambler's problem using the parameters of Examples 1-4.

\begin{tabular}{ccccc}
\hline$p$ & $x_{1}$ & $x_{2}$ & $x_{3}$ & Optimal utility \\
\hline \multicolumn{5}{c}{$f(y)=\log (a+x), B=1000, a=10, n=2$} \\
\hline 0.5 & 500.0 & 500.0 & 500.0 & 5.4 \\
0.6 & 500.0 & 500.0 & 500.0 & 6.1 \\
0.7 & 540.0 & 620.0 & 460.0 & 6.6 \\
0.8 & 669.2 & 1007.5 & 330.9 & 7.1 \\
0.9 & 821.8 & 1465.5 & 178.2 & 7.6 \\
\hline \multicolumn{5}{c}{$f(y)=y^{\alpha}, B=1000, \alpha=0.5, n=2$} \\
\hline 0.5 & 500.0 & 500.0 & 500.0 & 27.0 \\
0.6 & 529.4 & 588.2 & 470.6 & 31.3 \\
0.7 & 731.3 & 1194.0 & 268.7 & 36.2 \\
0.8 & 888.9 & 1666.7 & 111.1 & 42.9 \\
0.9 & 975.9 & 1927.7 & 24.1 & 51.9 \\
\hline$f(y)=-\mathrm{e}^{-b y}, B=1000, b=0.001, n=2$ \\
\hline 0.5 & 500.0 & 500.0 & 500.0 & -0.22 \\
0.6 & 500.0 & 500.0 & 500.0 & -0.22 \\
0.7 & 500.0 & 500.0 & 500.0 & -0.22 \\
0.8 & 564.4 & 693.2 & 435.6 & -0.20 \\
0.9 & 699.5 & 1098.6 & 300.5 & -0.15 \\
\hline$f(y)=y-c y^{2}, B=1000, c=0.00005, n=2$ \\
\hline 0.5 & 500 & 500 & 500 & 925 \\
0.6 & 875 & 1625 & 125 & 1158 \\
0.7 & 1000 & 2000 & 0 & 1568 \\
0.8 & 1000 & 2000 & 0 & 2048 \\
0.9 & 1000 & 2000 & 0 & 2592 \\
\hline \multicolumn{5}{c}{}
\end{tabular}


TABLE 2: Optimal solutions to the action gambler's problem using the parameters of Examples 1-4.

\begin{tabular}{|c|c|c|c|c|c|c|c|c|}
\hline$p$ & $x_{1}$ & $x_{2}$ & $x_{3}$ & $x_{4}$ & $x_{5}$ & $x_{6}$ & $x_{7}$ & Optimal utility \\
\hline \multicolumn{9}{|c|}{$f(y)=\log (a+x), B=1000, a=10, n=3$} \\
\hline 0.5 & 333.3 & 333.3 & 333.3 & 333.3 & 333.3 & 333.3 & 333.3 & 6.1 \\
\hline 0.6 & 333.3 & 333.3 & 333.3 & 333.3 & 333.3 & 333.3 & 333.3 & 6.6 \\
\hline 0.7 & 441.4 & 580.6 & 302.3 & 812.8 & 348.4 & 348.4 & 256.3 & 7.0 \\
\hline 0.8 & 621.2 & 1015.3 & 253.6 & 1545.6 & 334.4 & 370.3 & 125.3 & 7.5 \\
\hline 0.9 & 667.7 & 1129.3 & 285.7 & 1063.1 & 477.0 & 355.6 & 46.6 & 7.9 \\
\hline \multicolumn{9}{|c|}{$f(y)=y^{\alpha}, B=1000, \alpha=0.5, n=3$} \\
\hline 0.5 & 333.3 & 333.3 & 333.3 & 333.3 & 333.3 & 333.3 & 333.3 & 29.0 \\
\hline 0.6 & 426.2 & 548.5 & 303.8 & 759.5 & 337.6 & 337.6 & 270.0 & 33.0 \\
\hline 0.7 & 696.0 & 1169.7 & 222.3 & 1976.3 & 363.0 & 363.0 & 81.7 & 39.4 \\
\hline 0.8 & 882.7 & 1661.2 & 104.2 & 3127.0 & 195.4 & 195.4 & 13.0 & 50.2 \\
\hline 0.9 & 975.6 & 1927.4 & 23.8 & 3807.8 & 47.0 & 47.0 & 0.6 & 66.4 \\
\hline \multicolumn{9}{|c|}{$f(y)=-\mathrm{e}^{-b y}, B=1000, b=0.001, n=3$} \\
\hline 0.5 & 333.3 & 333.3 & 333.3 & 333.3 & 333.3 & 333.3 & 333.3 & -0.31 \\
\hline 0.6 & 333.3 & 333.3 & 333.3 & 333.3 & 333.3 & 333.3 & 333.3 & -0.29 \\
\hline 0.7 & 385.0 & 423.7 & 346.3 & 423.7 & 423.8 & 423.8 & 268.8 & -0.26 \\
\hline 0.8 & 538.9 & 693.2 & 384.7 & 693.2 & 693.1 & 693.1 & 76.4 & -0.19 \\
\hline 0.9 & 636.5 & 912.0 & 363.5 & 1096.3 & 724.5 & 727.1 & 0.0 & -0.10 \\
\hline \multicolumn{9}{|c|}{$f(y)=y-c y^{2}, B=1000, c=0.00005, n=3$} \\
\hline 0.5 & 333.3 & 333.3 & 333.3 & 333.3 & 333.3 & 333.3 & 333.3 & 933.3 \\
\hline 0.6 & 681.8 & 1045.5 & 318.2 & 1454.6 & 636.4 & 636.4 & 0.0 & 1229.2 \\
\hline 0.7 & 833.3 & 1500.0 & 166.7 & 2666.7 & 333.3 & 333.3 & 0.0 & 1724.8 \\
\hline 0.8 & 961.5 & 1884.6 & 38.5 & 3692.3 & 76.9 & 76.9 & 0.0 & 2463.5 \\
\hline 0.9 & 1000.0 & 2000.0 & 0.0 & 4000.0 & 0.0 & 0.0 & 0.0 & 3499.2 \\
\hline
\end{tabular}

(iii) When $n=2, x_{1}+x_{3}=1000$ for all $p$. When $n=3, x_{1}+x_{3}+x_{7}=1000$ for all $p$.

(iv) The wagers on the first bet are nondecreasing as $p$ increases.

\section{A related result}

In this brief section we state a generalization of Theorem 1 that holds for the generalized action gambler's problem where the gambler is required to wager, in total, an amount $A \leq B$, where $B$ is the initial bankroll. We also make a few observations about this result. The proof is given at the end of Subsection 6.1 as a slight variation of the proof of Theorem 1.

Theorem 3. Let $f(y)$ be a concave, increasing, and differentiable utility function on $[0, M]$, let $n \geq 1$, and let $A<B$. Then, for the generalized action gambler's problem, wagering $A / n$ on each bet maximizes the expected utility of the final bankroll if and only if

$$
p \leq \min _{\omega \in\{1, \ldots, n\}}\left(1+\frac{\mathrm{d}}{\mathrm{d} \omega} f\left((B-A)+\frac{2 A}{n} \omega\right) / \frac{\mathrm{d}}{\mathrm{d} \omega} f\left((B-A)+\frac{2 A}{n}(\omega-1)\right)\right)^{-1} .
$$

Next we consider some implications of the theorem. 
First, observe that, when $A=0$, the upper bound on $p$ is $\frac{1}{2}$. This means that it is optimal to wager nothing when the bets have nonpositive expected value.

Next, let $p^{*}$ denote the upper bound on $p$ given in the theorem. Observe that there can be a discontinuity in the value of $p^{*}$ as $A \rightarrow B$. This is suggested by the range of values for $\omega$, which is different for the cases in which $A<B$ and $A=B$ when $n \geq 2$. But, it can also be seen for the following example: consider the case in which $n=1$ and the utility function $f(x)=\log (a+x)$ for $a>0$. Without the action constraint, there is a value

$$
p^{* *}=\frac{a+2 B}{2 a+2 B}
$$

such that, for $p>p^{* *}$, it is optimal to wager $B$ (see [3]). Now consider adding the action constraint. When $A=B$, we have $p^{*}=1$ (i.e. it is always optimal to wager $A / 1$ ). But, if $A<B$ then wagering $A$ is not optimal for $p \in\left(p^{* *}, 1\right]$. So we have $p^{*} \leq p^{* *}$ when $A<B$ and $p^{*}=1$ when $A=B$.

The theorem also implies the following.

Remark 6. Suppose that $B$ is fixed. Let $p^{<}$denote the upper bound on $p$ in Theorem 3 when $A<B$, and let $p=$ denote the upper bound on $p$ in Theorem 1 when $A=B$. Then $p^{<} \leq p^{=}$ for all $0 \leq A \leq B$. Hence, if it is optimal to wager $A / n$ on each bet when $A<B$, then it is also optimal to wager $A / n$ on each bet when $A=B$.

With this remark in mind, consider a gambler for whom $A<B$ and $p$ is such that it is optimal to wager $A / n$ on each bet. In this case, for the optimal strategy, there is zero probability of losing the entire bankroll. For such a gambler, this may seem counter to the notion that a bankroll is the amount he wants to risk. Hence, he may want to reset $A$ to equal $B$. The remark says that it will then be optimal to wager $B / n$ on each bet. This is why we emphasize the case in which $A=B$ in this paper.

\section{Proofs}

In this section we prove Theorems 1 and 3, and Proposition 1. The main technique used in our proofs is an application of the well-known Karush-Kuhn-Tucker conditions to the nonlinear programming formulation given in Subsection 4.1.

\subsection{Proof of Theorems 1 and 3}

We first prove Theorem 1 . We then discuss the slight modifications necessary for proving Theorem 3.

Let us begin by rewriting the nonlinear program in Subsection 4.1 as follows:

$$
\max \sum_{v \in \operatorname{Leaves}(T)} p^{w(v)}(1-p)^{l(v)} f\left(B+d^{v} x\right)
$$

such that

$$
\begin{gathered}
-B-d^{v} x+x_{v} \leq 0 \quad \text { for all } v \in \operatorname{NonLeaves}(T) \backslash v^{L} \\
B-e^{v} x \leq 0 \quad \text { for all } v \in \operatorname{NearLeaves}(T) \backslash v^{L} \\
B-e^{v^{L}} x=0 \\
-x \leq 0
\end{gathered}
$$


Let us rewrite this nonlinear program again as follows, where the new functions are defined with the obvious correspondences to the above system. Call the following program $S^{\prime}$ :

$$
\max F(x)
$$

such that

$$
\begin{gathered}
G^{v}(x) \leq 0 \quad \text { for all } v \in \operatorname{NonLeaves}(T) \backslash v^{L}, \\
\bar{G}^{v}(x) \leq 0 \quad \text { for all } v \in \operatorname{NearLeaves}(T) \backslash v^{L}, \\
H(x)=0, \\
\widetilde{G}^{v}(x) \leq 0 \quad \text { for all } v \in \operatorname{NonLeaves}(T) .
\end{gathered}
$$

Let us consider, for $S^{\prime}$, the feasible vector $x^{*}$ defined by $x_{i}^{*}=B / n$ for all $i \in \operatorname{NonLeaves}(T)$. Associate the following variables with each constraint in $S^{\prime}: \mu_{v}$ with each constraint in (7); $\bar{\mu}_{v}$ with each constraint in (8); $\mu_{H}$ with the constraint (9); and $\tilde{\mu}_{v}$ with each constraint in (10). Because the objective function is concave and the constraint functions are convex for $S^{\prime}$, the theory of constrained nonlinear programming (see, e.g. [26, pp. 385-400]) tells us the following. The vector $x^{*}$ is a global maximum for $S^{\prime}$ if and only if the following system $S$ (determined by the Karush-Kuhn-Tucker conditions) evaluated at $x=x^{*}$ has a solution:

$$
\begin{gathered}
\frac{\partial}{\partial x_{i}} F(x)+\sum_{v \in \operatorname{NonLeaves}(T) \backslash v^{L}} \mu_{v} \frac{\partial}{\partial x_{i}} G^{v}(x)+\sum_{v \in \operatorname{NearLeaves}(T) \backslash v^{L}} \bar{\mu}_{v} \frac{\partial}{\partial x_{i}} \bar{G}^{v}(x) \\
+\mu_{H} \frac{\partial}{\partial x_{i}} H(x)+\sum_{v \in \operatorname{NonLeaves}(T)} \tilde{\mu}_{v} \frac{\partial}{\partial x_{i}} \widetilde{G}^{v}(x) \\
=0 \quad \text { for all } i \in \operatorname{NonLeaves}(T), \\
\mu_{v} G^{v}(x)=0 \text { for all } v \in \operatorname{NonLeaves}(T) \backslash v^{L}, \\
\bar{\mu}_{v} \bar{G}^{v}(x)=0 \text { for all } v \in \operatorname{NearLeaves}(T) \backslash v^{L}, \\
\tilde{\mu}_{v} \widetilde{G}^{v}(x)=0 \quad \text { for all } v \in \operatorname{NonLeaves}(T), \\
\mu_{v} \leq 0 \quad \text { for all } v \in \operatorname{NonLeaves}(T) \backslash v^{L}, \\
\bar{\mu}_{v} \leq 0 \quad \text { for all } v \in \operatorname{NearLeaves}(T) \backslash v^{L} \\
\tilde{\mu}_{v} \leq 0 \text { for all } v \in \operatorname{NonLeaves}(T) .
\end{gathered}
$$

Observe that

- $G^{v}\left(x^{*}\right)<0$, which implies that $\mu_{v}=0$ for all $v \in \operatorname{NonLeaves}(T) \backslash v^{L}$; and

- $\widetilde{G}^{v}\left(x^{*}\right)<0$, which implies that $\tilde{\mu}_{v}=0$ for all $v \in \operatorname{NonLeaves}(T)$.

Also, $\bar{G}^{v}\left(x^{*}\right)=0$ for all $v \in \operatorname{NearLeaves}(T) \backslash v^{L}$; hence, we can ignore the constraints in (11).

Finally, if we let $\bar{\mu}_{v}$ and $\bar{G}^{v}(x)$ be defined at $v=v^{L}$, in the obvious way, then we have $H(x)=\bar{G}^{v^{L}}(x)$. So, we can simplify the system as follows:

$$
\begin{gathered}
\frac{\partial}{\partial x_{i}} F(x)+\sum_{v \in \operatorname{NearLeaves}(T)} \bar{\mu}_{v} \frac{\partial}{\partial x_{i}} \bar{G}^{v}(x)=0 \text { for all } i \in \operatorname{NonLeaves}(T), \\
\bar{\mu}_{v} \leq 0 \quad \text { for all } v \in \operatorname{NearLeaves}(T) \backslash v^{L}
\end{gathered}
$$


Let us perform a convenient change of variables, and let $y_{v}=B+d^{v} x$. Then, substituting for $F(x)$ and $y_{v}$, and evaluating $\partial \bar{G}^{v}(x) / \partial x_{i}$, we can rewrite the system as follows:

$$
\begin{gathered}
\sum_{s \in \text { Leaves }(i)} p^{w(s)}(1-p)^{l(s)} \frac{\mathrm{d}}{\mathrm{d} y_{s}} f\left(y_{s}\right) \frac{\partial}{\partial x_{i}} y_{s} \\
=\sum_{v \in \operatorname{NearLeaves}(i)} \bar{\mu}_{v} \text { for all } i \in \operatorname{NonLeaves}(T), \\
\bar{\mu}_{v} \leq 0 \text { for all } v \in \operatorname{NearLeaves}(T) \backslash v^{L} .
\end{gathered}
$$

Consider equality (12) for the special case in which $i \in \operatorname{NearLeaves}(T)$. In this case the equality has only one variable $\bar{\mu}_{v}$ and $|\operatorname{Leaves}(i)|=2$. We define

$$
\bar{\mu}_{i}^{*}=\sum_{s \in \operatorname{Leaves}(i)} p^{w(s)}(1-p)^{l(s)} \frac{\mathrm{d}}{\mathrm{d} y_{s}} f\left(y_{s}\right) \frac{\partial}{\partial x_{\operatorname{Pred}(s)}} y_{s} \quad \text { for all } i \in \operatorname{NearLeaves}(T) .
$$

(Note the use of the Pred(.) function.) We now substitute the values for $\bar{\mu}_{i}^{*}$ when $i \in$ NearLeaves $(T)$ into (12) to obtain

$$
\begin{aligned}
& \sum_{s \in \text { Leaves }(i)} p^{w(s)}(1-p)^{l(s)} \frac{\mathrm{d}}{\mathrm{d} y_{s}} f\left(y_{s}\right) \frac{\partial}{\partial x_{i}} y_{s} \\
& \quad=\sum_{s \in \operatorname{Leaves}(i)} p^{w(s)}(1-p)^{l(s)} \frac{\mathrm{d}}{\mathrm{d} y_{s}} f\left(y_{s}\right) \frac{\partial}{\partial x_{\operatorname{Pred}(s)}} y_{s} \quad \text { for all } i \in \operatorname{NonLeaves}(T) .
\end{aligned}
$$

Claim 1. Equality (14) holds.

Proof. Pick any $i \in \operatorname{NonLeaves}(T)$ and consider the corresponding equality. Observe that both sides of the equality are identical, except for the partial derivatives. Note that $w(s) \in$ $\{0,1, \ldots . n\}$. We show that, for any value $j \in\{0,1, \ldots, n\}$, the sum of the terms on the lefthand side with $w(s)=j$ equals the sum of the terms on the right-hand side with $w(s)=j$. Observe that the partial derivatives are all equal to +1 or -1 . Hence, if we can show, for fixed $j$, that the number of partial derivatives on the left-hand side that equal +1 is equal to the number of partial derivatives on the right-hand side that equal +1 , we are done.

Consider the set of directed paths, call it $P$, in $T$ from $i$ to a node in Leaves $(i)$ such that the (unique) extension of this path to $r$ contains exactly $j$ wins. The endnodes of these paths are precisely the nodes in Leaves $(i)$ such that $w(s)=j$. If node $i$ corresponds to bet $k$ then the paths in $P$ correspond to all the permutations of $n-k+1$ wins and losses. Hence, the total number of wins in any position of these permutations is the same. Finally, observe that the number of partial derivatives on the left-hand side with value +1 is equal to the number of wins in the first position of these permutations, and that the number of partial derivatives on the right-hand side with value +1 is equal to the number of wins in the last position of these permutations. The result follows.

It now follows that, for $x^{*}$, the vector $\bar{\mu}^{*}$ is the unique solution to the equalities in our system. It remains to determine conditions under which $\bar{\mu}_{v}^{*} \leq 0$ for all $v \in \operatorname{NearLeaves}(T) \backslash v^{L}$.

Let $v \in \operatorname{NearLeaves}(T) \backslash v^{L}$, and let $v^{\prime}$ and $v^{\prime \prime}$ be the two nodes in Leaves $(v)$ corresponding to a win and a loss, respectively. It is easy to check that, in (13),

$$
\frac{\partial}{\partial x_{\operatorname{Pred}\left(v^{\prime}\right)}} y_{v^{\prime}}=+1 \text { and } \frac{\partial}{\partial x_{\operatorname{Pred}\left(v^{\prime \prime}\right)}} y_{v^{\prime \prime}}=-1 .
$$


D. HARTVIGSEN

In addition, we have $w\left(v^{\prime}\right)=w\left(v^{\prime \prime}\right)+1$ and $l\left(v^{\prime}\right)=l\left(v^{\prime \prime}\right)-1$. Thus, (13) becomes

$$
\bar{\mu}_{v}^{*}=p^{w\left(v^{\prime}\right)}(1-p)^{l\left(v^{\prime}\right)} \frac{\mathrm{d}}{\mathrm{d} y_{v^{\prime}}} f\left(y_{v^{\prime}}\right)-p^{w\left(v^{\prime}\right)-1}(1-p)^{l\left(v^{\prime}\right)+1} \frac{\mathrm{d}}{\mathrm{d} y_{v^{\prime \prime}}} f\left(y_{v^{\prime \prime}}\right)
$$

for all $v \in \operatorname{NearLeaves}(T) \backslash v^{L}$. It follows that conditions for which $\bar{\mu}_{v}^{*} \leq 0$ are determined by conditions for which

$$
p \frac{\mathrm{d}}{\mathrm{d} y_{v^{\prime}}} f\left(y_{v^{\prime}}\right)-(1-p) \frac{\mathrm{d}}{\mathrm{d} y_{v^{\prime \prime}}} f\left(y_{v^{\prime \prime}}\right) \leq 0 \quad \text { for all } v \in \operatorname{NearLeaves}(T) \backslash v^{L}
$$

or

$$
p \leq\left(1+\frac{\mathrm{d}}{\mathrm{d} y_{v^{\prime}}} f\left(y_{v^{\prime}}\right) / \frac{\mathrm{d}}{\mathrm{d} y_{v^{\prime \prime}}} f\left(y_{v^{\prime \prime}}\right)\right)^{-1} \text { for all } v \in \operatorname{NearLeaves}(T) \backslash v^{L} .
$$

Observe that, because $f$ is concave, increasing, and differentiable, we are not dividing by 0 in this expression. Because this expression is being evaluated at $x^{*}$, we know that

$$
y_{v^{\prime}}=B+\frac{B}{n} w\left(v^{\prime}\right)-\frac{B}{n}\left(n-w\left(v^{\prime}\right)\right) \quad \text { for all } v \in \operatorname{NearLeaves}(T) \backslash v^{L}
$$

or

$$
y_{v^{\prime}}=\frac{2 B}{n} w\left(v^{\prime}\right) \quad \text { for all } v \in \operatorname{NearLeaves}(T) \backslash v^{L}
$$

and

$$
y_{v^{\prime \prime}}=B+\frac{B}{n}\left(w\left(v^{\prime}\right)-1\right)-\frac{B}{n}\left(n-w\left(v^{\prime}\right)+1\right) \quad \text { for all } v \in \operatorname{NearLeaves}(T) \backslash v^{L}
$$

or

$$
y_{v^{\prime \prime}}=\frac{2 B}{n}\left(w\left(v^{\prime}\right)-1\right) \quad \text { for all } v \in \operatorname{NearLeaves}(T) \backslash v^{L} .
$$

Because $v \in$ NearLeaves $(T) \backslash v^{L}$, we have $w\left(v^{\prime}\right) \in\{2, \ldots, n\}$. So, using these expressions for $y_{v^{\prime}}$ and $y_{v^{\prime \prime}}$, and setting $\omega=w\left(v^{\prime}\right),(15)$ can be rewritten as

$$
p \leq\left(1+\left.\frac{\mathrm{d}}{\mathrm{d} y} f(y)\right|_{2 B \omega / n} /\left.\frac{\mathrm{d}}{\mathrm{d} y} f(y)\right|_{2 B(\omega-1) / n}\right)^{-1} \text { for } \omega \in\{2, \ldots, n\} .
$$

Finally, applying the chain rule, this can be rewritten as

$$
p \leq\left(1+\frac{\mathrm{d}}{\mathrm{d} \omega} f\left(\frac{2 B}{n} \omega\right) / \frac{\mathrm{d}}{\mathrm{d} \omega} f\left(\frac{2 B}{n}(\omega-1)\right)\right)^{-1} \text { for } \omega \in\{2, \ldots, n\} .
$$

The result now follows.

Let us finally consider the proof of Theorem 3 . We begin with the system

$$
\max \sum_{v \in \operatorname{Leaves}(T)} p^{w(v)}(1-p)^{l(v)} f\left(B+d^{v} x\right)
$$

such that

$$
\begin{gathered}
-B-d^{v} x+x_{v} \leq 0 \quad \text { for all } v \in \operatorname{NonLeaves}(T) \\
A-e^{v} x \leq 0 \quad \text { for all } v \in \operatorname{NearLeaves}(T) \\
-x \leq 0
\end{gathered}
$$


and rewrite it as

$\max F(x)$

such that

$$
\begin{array}{cc}
G^{v}(x) \leq 0 & \text { for all } v \in \operatorname{NonLeaves}(T), \\
\bar{G}^{v}(x) \leq 0 & \text { for all } v \in \operatorname{NearLeaves}(T), \\
\widetilde{G}^{v}(x) \leq 0 & \text { for all } v \in \operatorname{NonLeaves}(T)
\end{array}
$$

The proof now is essentially identical to the proof of Theorem 1 with the following exceptions.

- Let $x^{*}$ be defined by $x_{i}^{*}=A / n$ for all $i \in \operatorname{NonLeaves}(T)$.

- The term involving $H(x)$ in system $S$ is removed.

- The sentence after the statement of $S$ beginning with 'Finally, if we let $\bar{\mu}_{v} \ldots$ '. is removed.

- All occurrences of ' $\backslash v^{L}$ ' are removed.

- Equations (16)-(19) become

$$
y_{v^{\prime}}=B+\frac{A}{n} w\left(v^{\prime}\right)-\frac{A}{n}\left(n-w\left(v^{\prime}\right)\right) \quad \text { for all } v \in \operatorname{NearLeaves}(T)
$$

or

$$
y_{v^{\prime}}=(B-A)+\frac{2 A}{n} w\left(v^{\prime}\right) \quad \text { for all } v \in \operatorname{NearLeaves}(T)
$$

and

$$
y_{v^{\prime \prime}}=B+\frac{A}{n}\left(w\left(v^{\prime}\right)-1\right)-\frac{A}{n}\left(n-w\left(v^{\prime}\right)+1\right) \quad \text { for all } v \in \operatorname{NearLeaves}(T)
$$

or

$$
y_{v^{\prime \prime}}=(B-A)+\frac{2 A}{n}\left(w\left(v^{\prime}\right)-1\right) \quad \text { for all } v \in \operatorname{NearLeaves}(T) .
$$

- Occurrences of $\omega \in\{2, \ldots, n\}$ in the bounds must be changed to $\omega \in\{1, \ldots, n\}$.

\subsection{Proof of Theorem 2}

The proof we present is a modified and simpler version of the proof of Theorem 1 .

Consider the following version of the nonlinear program from the previous section:

$$
\max \sum_{v \in \operatorname{Leaves}(T)} p^{w(v)}(1-p)^{l(v)} f\left(B+d^{v} x\right)
$$

such that

$$
\begin{gathered}
B-e^{v} x=0 \quad \text { for all } v \in \operatorname{NearLeaves}(T), \\
-x \leq 0 .
\end{gathered}
$$

The objective function is the same. However, the nontrivial constraints say that the gambler must always wager a total amount of exactly $B$. The problem described by this formulation is a relaxation of the simultaneous gambling problem. In particular, we have imposed an order on the bets and each bet, after the first, has more than one variable associated with it. So, to capture the simultaneous gambling problem, we need to add a constraint for each bet requiring 
that the associated variables are all equal. However, if we can prove that setting each variable to $B / n$ is optimal for the above problem, then, since that solution is feasible for the simultaneous gambling problem, it must also be optimal for that problem.

Let us rewrite this system as follows, where the new functions are defined with the obvious correspondence to the above system:

$$
\max F(x)
$$

such that

$$
\begin{array}{cc}
\bar{G}^{v}(x)=0 & \text { for all } v \in \operatorname{NearLeaves}(T), \\
\widetilde{G}^{v}(x) \leq 0 & \text { for all } v \in \operatorname{NonLeaves}(T) .
\end{array}
$$

As before, let $x^{*}$ denote the feasible vector defined by $x_{i}^{*}=B / n$ for all $i \in \operatorname{NonLeaves}(T)$. Let us associate the following variables with each constraint in the above system: $\bar{\mu}_{v}$ with each constraint in (20) and $\tilde{\mu}_{v}$ with each constraint in (21). As before, the theory of nonlinear programming tells us that $x^{*}$ is the global maximum for the above nonlinear program if and only if the following system, evaluated at $x=x^{*}$, has a solution:

$$
\frac{\partial}{\partial x_{i}} F(x)+\sum_{v \in \operatorname{NearLeaves}(T)} \bar{\mu}_{v} \frac{\partial}{\partial x_{i}} \bar{G}^{v}(x)+\sum_{v \in \operatorname{NonLeaves}(T)} \tilde{\mu}_{v} \frac{\partial}{\partial x_{i}} \widetilde{G}^{v}(x)=0
$$

for all $i \in \operatorname{NonLeaves}(T)$,

$$
\begin{gathered}
\tilde{\mu}_{v} \widetilde{G}^{v}(x)=0 \text { for all } v \in \operatorname{NonLeaves}(T), \\
\tilde{\mu}_{v} \leq 0 \text { for all } v \in \operatorname{NonLeaves}(T)
\end{gathered}
$$

Observe that $\widetilde{G}^{v}\left(x^{*}\right)<0$, which implies that $\tilde{\mu}_{v}=0$ for all $v \in \operatorname{NonLeaves}(T)$. So, we can simplify the system as follows:

$$
\frac{\partial}{\partial x_{i}} F(x)+\sum_{v \in \operatorname{NearLeaves}(T)} \bar{\mu}_{v} \frac{\partial}{\partial x_{i}} \bar{G}^{v}(x)=0 \quad \text { for all } i \in \operatorname{NonLeaves}(T) .
$$

Let us perform a convenient change of variables, and let $y_{v}=B+d^{v} x$. Then, substituting for $F(x)$ and $y_{v}$, and evaluating $\partial \bar{G}^{v}(x) / \partial x_{i}$, we can rewrite the system as follows:

$$
\sum_{s \in \operatorname{Leaves}(i)} p^{w(s)}(1-p)^{l(s)} \frac{\mathrm{d}}{\mathrm{d} y_{s}} f\left(y_{s}\right) \frac{\partial}{\partial x_{i}} y_{s}=\sum_{v \in \operatorname{NearLeaves}(i)} \bar{\mu}_{v} \quad \text { for all } i \in \operatorname{NonLeaves}(T) .
$$

We now proceed exactly as in the proof of Theorem 1 and show that $\bar{\mu}_{v}^{*}$ (as defined above) is the unique solution to (22). Because $\bar{\mu}_{v}^{*}$ is unrestricted in this model, the result follows for all values of $p$.

\subsection{Proof of Proposition 1}

Let us define the function $g:[2, n] \rightarrow \mathbb{R}$ as follows:

$$
g(\omega)=\frac{\mathrm{d}}{\mathrm{d} \omega} f\left(\frac{2 B}{n} \omega\right) / \frac{\mathrm{d}}{\mathrm{d} \omega} f\left(\frac{2 B}{n}(\omega-1)\right) .
$$


Observe that the minimum in (1) is achieved at $\omega=n$ if $\mathrm{d} g(\omega) / \mathrm{d} \omega>0$ for $\omega \in[2, n]$; it is achieved at $\omega=2$ if $\mathrm{d} g(\omega) / \mathrm{d} \omega<0$ for $\omega \in[2, n]$; and it is achieved at all points in [2,n] if $\mathrm{d} g(\omega) / \mathrm{d} \omega=0$ for $\omega \in[2, n]$. Let us compute $\mathrm{d} g(\omega) / \mathrm{d} \omega$ for $\omega \in[2, n]$ :

$$
\begin{aligned}
\frac{\mathrm{d}}{\mathrm{d} \omega} g(\omega)= & \left(\frac{\mathrm{d}}{\mathrm{d} \omega} f\left(\frac{2 B}{n}(\omega-1)\right) \frac{\mathrm{d}^{2}}{\mathrm{~d} \omega^{2}} f\left(\frac{2 B}{n} \omega\right)-\frac{\mathrm{d}}{\mathrm{d} \omega} f\left(\frac{2 B}{n} \omega\right) \frac{\mathrm{d}^{2}}{\mathrm{~d} \omega^{2}} f\left(\frac{2 B}{n}(\omega-1)\right)\right) \\
& \times\left(\frac{\mathrm{d}}{\mathrm{d} \omega} f\left(\frac{2 B}{n}(\omega-1)\right)\right)^{-2} .
\end{aligned}
$$

Since we are only interested in the sign of this expression, we can ignore the denominator. Furthermore, since $\mathrm{d} f(2 B \omega / n) / \mathrm{d} \omega>0$ and $\mathrm{d} f(2 B(\omega-1) / n) / \mathrm{d} \omega>0$ on $[2, n]$ (because $f$ is increasing for $\omega \in[2, n])$, we can divide the above expression by

$$
\frac{\mathrm{d}}{\mathrm{d} \omega} f\left(\frac{2 B}{n} \omega\right) \frac{\mathrm{d}}{\mathrm{d} \omega} f\left(\frac{2 B}{n}(\omega-1)\right)
$$

without changing its sign. We can then relate the resulting expression to the Arrow-Pratt absolute risk aversion coefficient to obtain the following:

$$
\begin{aligned}
& h\left(\frac{2 B}{n}(\omega-1)\right)-h\left(\frac{2 B}{n}(\omega)\right) \\
& \quad=\frac{\mathrm{d}^{2}}{\mathrm{~d} \omega^{2}} f\left(\frac{2 B}{n} \omega\right) / \frac{\mathrm{d}}{\mathrm{d} \omega} f\left(\frac{2 B}{n} \omega\right)-\frac{\mathrm{d}^{2}}{\mathrm{~d} \omega^{2}} f\left(\frac{2 B}{n}(\omega-1)\right) / \frac{\mathrm{d}}{\mathrm{d} \omega} f\left(\frac{2 B}{n}(\omega-1)\right) .
\end{aligned}
$$

So, if $h(y)$ is decreasing as $y$ increases on $[2 B / n, 2 B]$ then $\mathrm{d} g(\omega) / \mathrm{d} \omega>0$ for $\omega \in[2, n]$; if $h(y)$ is increasing as $y$ increases on $[2 B / n, 2 B]$ then $\mathrm{d} g(\omega) / \mathrm{d} \omega<0$ for $\omega \in[2, n]$; and if $h(y)$ is constant on $[2 B / n, 2 B]$ then $\mathrm{d} g(\omega) / \mathrm{d} \omega=0$ for $\omega \in[2, n]$. The result now follows.

\section{An open problem}

We have studied the action gambler's problem for general utility functions and applied the general results to some specific examples. An open problem is to find a closed-form solution to the action gambler's problem for a specific common utility function for all values of $p$. This was done in [3] for the unconstrained version of the problem for the utility function $f(y)=\log (a+y), a \geq 0$. This particular function is interesting because it is a generalization of the $f(y)=\log (y)$ function used in [15], but is defined at 0 , when $a>0$, as it is necessary for the constrained problem. Another possibly tractable candidate is $f(y)=y^{\alpha}$ for $0<\alpha<1$.

\section{References}

[1] Bellman, R. and Kalaba, R. (1957). Dynamic programming and statistical communication theory. Proc. Nat. Acad. Sci. USA 43, 749-751.

[2] Breiman, L. (1961). Optimal gambling systems for favorable games. In Proc. 4th Berkeley Symp. Math. Statist. Prob., Vol. I, University of California Press, Berkeley, pp. 65-78.

[3] Cetinkaya, S. and Parlar, M. (1997). Optimal nonmyopic gambling strategy for the generalized Kelly criterion. Naval Res. Logistics 44, 639-654.

[4] Chen, R. W., Shepp, L. A., Yao, Y.-C. and Zhang, C.-H. (2005). On optimality of bold play for primitive casinos in the presence of inflation. J. Appl. Prob. 42, 121-137.

[5] DeGroot, M. H. (2004). Optimal Statistical Decisions. John Wiley, Hoboken, NJ.

[6] Dubins, L. E. And Savage, L. J. (1976). Inequalities for Stochastic Processes (How to Gamble If You Must). Corrected republication of the 1965 edition. Dover, New York. 
[7] Ethier, S. N. And Levin, D. A. (2005). On the fundamental theorem of card counting, with application to the game of trente et quarante. Adv. Appl. Prob. 37, 90-107.

[8] Ethier, S. N. And TAvaré, S. (1983). The proportional bettor's return on investment. J. Appl. Prob. 20, 563-573.

[9] Ferguson, T. S. (1965). Betting systems which minimize the probability of ruin. J. SIAM 13, 795-818.

[10] Finkelstein, M. and Whitley, R. (1981). Optimal strategies for repeated games. Adv. Appl. Prob. 13, 415-428.

[11] Freedman, D. A. (1967). Timid play is optimal. Ann. Math. Statist. 38, 1281-1283.

[12] GuU, S.-M. AND WANG, S.-P. (2002). Optimal gambling strategy and relative risk aversion. J. Chinese Inst. Indust. Eng. 19, 34-40.

[13] Heath, D. C., Pruitt, W. E. And Sudderth, W. D. (1972). Subfair red-and-black with a limit. Proc. Amer. Math. Soc. 35, 555-560.

[14] KARUSH, W. (1939). Minima of functions of several variables with inequalities as side conditions. Masters Thesis, University of Chicago.

[15] Kelly, J. L., JR. (1956). A new interpretation of information rate. Bell. System Tech. J. 35, 917-926.

[16] Kunn, H. W. And Tucker, A. W. (1961). Nonlinear programming. In Proc. 2nd Berkeley Symp. Math. Statist. Prob., ed. J. Neyman, University of California Press, Berkeley, pp. 481-492.

[17] Kulldorff, M. (1993). Optimal control of favorable games with a time limit. SIAM J. Control Optimization 31, 52-69.

[18] Luenberger, D. G. (1998). Investment Science. Oxford University Press.

[19] Markowitz, H. M. (1952). Portfolio selection. J. Finance 7, 77-91.

[20] Molenafr, W. ANd van der Velde, E. A. (1967). How to survive a fixed number of fair bets. Ann. Math. Statist. 38, 1278-1280.

[21] Pemberton, M. and RaU, N. (2001). Mathematics for Economists: An Introductory Textbook. Manchester University Press.

[22] Ross, S. M. (1974). Dynamic programming and gambling models. Adv. Appl. Prob. 6, 593-606.

[23] Ruth, K. (1999). Favorable red and black on the integers with a minimum bet. J. Appl. Prob. 36, 837-851.

[24] Samuelson, P. A. (1971). The 'fallacy' of maximizing the geometric mean in long sequences of investing or gambling. Proc. Nat. Acad. Sci. USA 68, 2493-2496.

[25] Schweinsberg, J. (2005). Improving on bold play when the gambler is restricted. J. Appl. Prob. 42, 321-333.

[26] Sun, W. And Yuan, Y.-X. (2006). Optimization Theory and Methods. Springer, New York.

[27] Wolfram ReSEARCH. (2008). Mathematica 6. Champaign, IL.

[28] YaO, Y.-C. (2007). On optimality of bold play for discounted Dubins-Savage gambling problems with limited playing times. J. Appl. Prob. 44, 212-225. 